\title{
Acute methanol poisoning
}

\section{Intoxicação aguda por metanol}

Mauricio F. Villamar

A 24-year-old man presented with acute encephalopathy, hematemesis, and rigidity of the extremities. He had anion gap metabolic acidosis. Osmolar gap was normal. Plasma methanol level was $40 \mathrm{mg} / \mathrm{dL}$. The Figure shows his neuroimaging findings.

Treatment with fomepizole and hemodialysis improved his encephalopathy. No obvious visual deficits were noted. There was generalized hypertonia and hyperreflexia, and dystonia of the right arm.
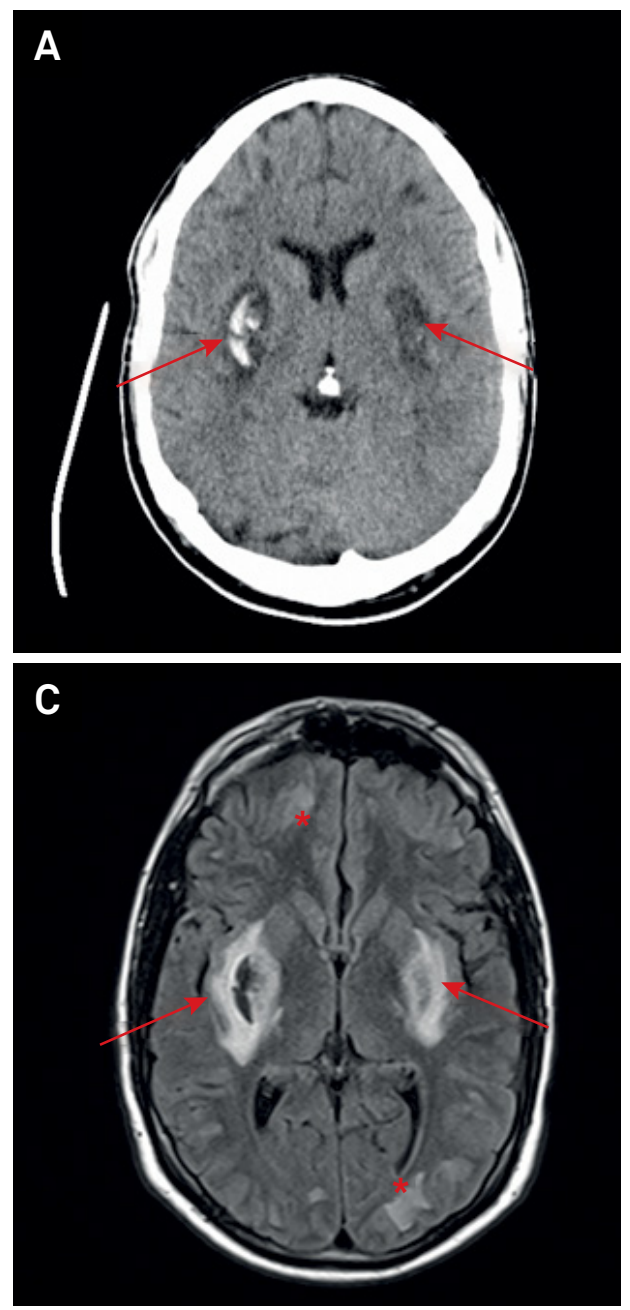

Formic acid, an end product of methanol metabolism, contributes to metabolic acidosis following methanol poisoning. Formate inhibits mitochondrial cytochrome oxidase, causing histotoxic hypoxia that preferentially affects the retina, optic nerve, subcortical white matter, and lentiform nucleus. Survivors may develop ophthalmologic abnormalities, dystonia or parkinsonism ${ }^{1,2,3}$.

Figure. Hemorrhagic necrosis of bilateral lentiform nuclei (arrows) was present on head CT (Panel A) and on non-contrast brain MRI (Panel B, T1-weighted; Panel C, T2/FLAIR-weighted; Panel D, susceptibility-weighted imaging). Subcortical white matter hyperintensities were also noted (Panel C, asterisks). 


\section{References}

1. Barceloux DG, Bond GR, Krenzelok EP, Cooper H, Vale JA. American Academy of Clinical Toxicology practice guidelines on the treatment of methanol poisoning. J Toxicol Clin Toxicol 2002;40(4):415-46. https://doi.org/10.1081/CLT-120006745

2. Giudicissi Filho M, Holanda CV, Nader NA, Gomes SR, Bertolucci PH. Bilateral putaminal hemorrhage related to methanol poisoning: a complication of hemodialysis? Case report. Arq Neuropsiquiatr 1995;53(3A):485-7. https://doi.org/10.1590/S0004-282X1995000300020

3. Kraut JA, Mullins ME. Toxic alcohols. N Engl J Med. 2018 Jan;378:270-80

https://doi.org/10.1056/NEJMra1615295 